\title{
Strings and Quantum Fields
}

\author{
Amit Giveon*† \\ Racah Institute of Physics, The Hebrew University, Jerusalem, 91904, Israel \\ E-mail: 'giveon@vms.hujicac.ili:
}

ABstract: We present a brief background on perturbative string theory, D-branes and the gauge theory on their worldvolume, solitonic NS5-branes and Little String Theory (LST) in their vicinity, and the holographic dualities relating strings propagating in the near horizon of such branes to theories without gravity, such as gauge theory or LST. We proceed by considering four dimensional string theories where the string scale is set at the electro-weak scale: $M_{s} \sim \mathrm{TeV}$. One possibility consists of theories on $1+$ 3 dimensional D-branes, where the gauge couplings are related to the string coupling, $g_{Y M}^{2} \sim g_{s}$, thus requiring large extra dimensions in order to set the correct Newton's constant $G \sim M_{P}^{-2}$. A very different possibility consists of string theory with a small coupling constant $g_{s} \sim M_{s} / M_{P} \sim 10^{-16}$, in which case the compactification scales can be set at a TeV. Charged particles are due to D-branes wrapped on small cycles in the compact six dimensional space, or in a dual picture to D-branes stretched between NS5branes. The gauge couplings are determined by geometric data - the two dimensional surface on which the fivebranes are wrapped - and thus can be set at the desired values independent of $g_{s}$. Such theories consist of low lying "Standard Model" particles coupled to neutral strings without gravity at a $\mathrm{TeV}-\mathrm{LST}$ at a $\mathrm{TeV}$.

\section{Background}

Consider string theory in flat ten dimensional space $R^{1,9}$, or in four dimensions $R^{1,3} \times \mathcal{M}_{6}$,

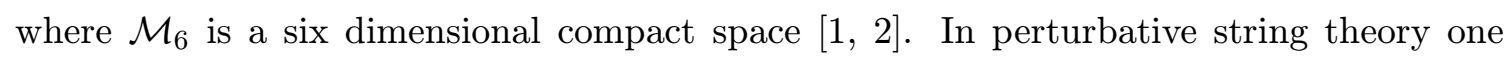
sums over all the worldsheets spanned by strings propagating in the ten dimensional target spacetime. The $n$ 'th order term in the string coupling $g_{s}$ expansion is a genus $n$ Riemann surface - a two dimensional worldsheet with $n$ "holes." Physical vertex operators $V$ on the worldsheet correspond in spacetime to on-shell observables $O_{V}\left(k_{\mu}^{2}=m^{2}\right)$. In particular, S-matrix elements in spacetime correspond to correlation functions on the worldsheet,

\footnotetext{
${ }^{*}$ Speaker.

${ }^{\dagger}$ Talk presented at the EPS-HEP, Budapest, 17 July 2001.
} 
summed over all genera and integrated over all worldsheet moduli:

$$
\sum_{n=0}^{\infty} g_{s}^{2 n-2} \int_{w . s .}\langle V \cdots V\rangle_{n} \quad \leftrightarrow \quad \text { S-matrix elements . }
$$

In addition to fundamental strings, string theory has solitonic objects which are $p$ dimensional hypersurfaces in ten dimensions. For instance, there are the so called Dbranes [i2]. At weak string coupling a D-brane is defined by the surface on which open strings can end. Let us discuss a $1+3$ dimensional D-brane, which we denote by D3. The low lying excitations of an open string ending on the D3-brane correspond to a photon. More precisely, the low energy theory on the D3-brane is the maximally supersymmetric Abelian gauge theory in four dimensions: a $U(1), N=4$ Super Yang-Mills (SYM). One can decouple this gauge theory from the higher string excitations, as well as gravity, by taking the limit in which the string tension $T_{s} \sim 1 / l_{s}^{2}$ is large: string excitations have masses of the order of the string scale $M_{s} \sim 1 / l_{s}$, which become infinitely massive in the limit $l_{s} \rightarrow 0$.

Consider now two parallel D3-branes ${ }^{1}$. The low lying excitations of the open strings stretched between one brane to the other correspond to charged gauge bosons in an $S U(2)$ gauge theory. The open string with one orientation corresponds say to the $W^{+}$while the one with the opposite orientation corresponds to $W^{-}$. In addition, there are D-strings which can stretch between the two D-branes; these correspond to magnetically charged particles in the four dimensional non-Abelian $N=4$ SYM.

String theory properties translate into gauge theory properties, and vice versa. In particular, the string coupling is related to the gauge coupling, and the distance between the D-branes correspond to the VEV of a scalar field in the adjoint of the gauge group, thus giving masses to charged particles. Non-trivial string phenomena translate into mysterious phenomena in gauge theories ${ }^{2}$. For instance, the so called S-duality in string theory interchanges fundamental strings with D-strings, and correspond to electric-magnetic, strong-weak coupling duality in gauge theory: $g_{s} \simeq g_{Y M}^{2} \leftrightarrow 1 / g_{s}$.

Another important solitonic object in string theory is the so called NS5-brane. This five dimensional object is the magnetic dual to a string in ten dimensions, pretty much like the magnetic dual of a particle in four dimensions is a particle. Consider a stack of NS5-branes. In the limit $g_{s} \rightarrow 0$ one obtains a $1+5$ dimensional theory $\left[\begin{array}{l}1 \\ 2\end{array}\right.$ of the open strings trapped in the vicinity of the fivebranes, as well as the D-branes which can stretch between the fivebranes. The strings in the vicinity of the fivebranes couple with strength $g_{s}$ to closed strings in the ten dimensional bulk, and thus decouple from them in the $g_{s} \rightarrow 0$ limit. On the contrary, the strings near the fivebranes couple strongly to each other, since the effective coupling near the fivebranes is large. This leads to a non-trivial interacting theory of strings without gravity, which is called Little String Theory (LST) [i].

LST has a single scale, the string scale $M_{s}$, and a Hagedorn spectrum of states with the Hagedorn temperature of the order of the string scale $T_{H} \sim M_{s}$. As the temperature approaches $T_{H}$ there is a phase transition, similar to QCD. We may compactify the

\footnotetext{
${ }^{1}$ Figures which follow the text can be found on the scanned transparencies in $3 . \overline{1}$.

${ }^{2}$ Though sometimes mysterious phenomena in one framework correspond to manifest properties of the other [üin].
} 
six dimensional LST to four dimensions by letting the fivebrane wrap a two dimensional compact cycle in the space $\mathcal{M}_{6}$ transverse to $R^{1,3}$. At low energy $E \ll M_{s}$ we have a four dimensional gauge theory with photons corresponding to the low lying excitations of closed strings in the vicinity of the fivebranes, and charged particles are due to the low lying excitations of D-branes stretched between the fivebranes.

The D-branes and NS5-branes are massive objects in a ten dimensional theory with gravity, and hence have back reaction on the background. The gravitational soliton due to the presence of a $p$ dimensional brane is a black $p$-brane, like a black hole. One may "magnify" the region in the vicinity of the branes, corresponding to looking at the near horizon of the gravitational black object. For instance, the near horizon background of a stack of D3-branes is a product of five dimensional Anti-de-Sitter space - a five dimensional,

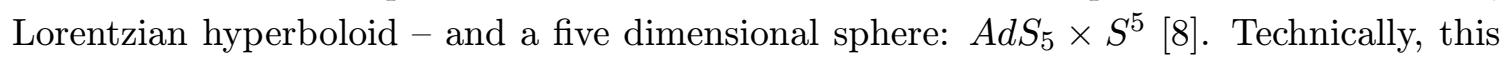
background can be obtained as the $l_{s} \rightarrow 0$ limit of the black 3-brane. Since this is the decoupling limit described above, it leads to the well celebrated conjecture [9. theory on $A d S_{5} \times S^{5}$ is equivalent to four dimensional $N=4 \mathrm{SYM}$ - a superconformal field theory (SCFT). This is a "holographic" duality, relating a five dimensional theory with gravity to a four dimensional theory without gravity. On-shell vertex operators $V$ on the worldsheet correspond in spacetime to observables $O_{V}$ in a four dimensional gauge theory. In particular, on-shell correlators on the worldsheet $\langle V \cdots V\rangle$ correspond to correlators $\left\langle O_{V} \cdots O_{V}\right\rangle$ in the four dimensional spacetime SCFT.

Similarly, the near horizon of a stack of NS5-branes correspond to the decoupling limit $g_{s} \rightarrow 0$, giving rise to LST described above [1] $\left.{ }_{1}^{1} \overline{0}\right]$. The gravitational background has an infinite "throat" - schematically, with a shape of a cylinder - along which the string coupling is varied. Down deep the throat, "close" to the fivebranes, the string coupling is large, while it becomes weaker as one goes away from the bottom of the throat. One can resolve the strong coupling singularity by "chopping" the infinite throat into a semiinfinite "cigar" shaped background [i] ${ }_{1}^{1} \overline{1}_{1}$. The little strings are associated with standard fundamental strings propagating in the cigar background, while charged degrees of freedom correspond to D-branes near the tip of the cigar. Hence we obtain another holographic duality: a theory with gravity in the cigar background is equivalent to a theory without gravity - LST in Minkowski space. On-shell observables in string theory on the cigar correspond to off-shell observables in LST. In particular, on-shell correlators $\langle V \cdots V\rangle$ on the worldsheet correspond to off-shell Green's functions $\left\langle O_{V}\left(k_{\mu}\right) \cdots O_{V}\left(k_{\mu}^{\prime}\right)\right\rangle$ in LST.

A richer class of interesting gauge theories and LSTs can be obtained in the decoupling

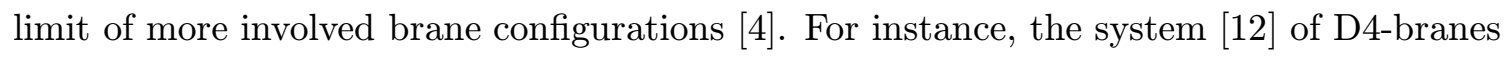
stretched between differently oriented NS5-branes gives rise to Supersymmetric QCD in

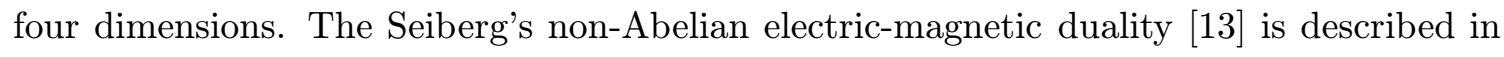
the brane picture by a smooth motion interchanging the location of the two fivebranes. In the near horizon limit of the fivebranes such SQCD is described by the dynamics of the low-lying excitations of open strings ending on D-branes located near the tip of the cigar background. 


\section{Strings and Branes at a TeV}

Between 1985 till 1995, the phenomenologically interesting string theories were mainly

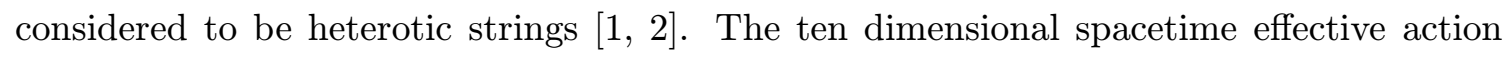
takes the schematic form:

$$
S_{e f f}^{(10)} \sim \frac{M_{s}^{8}}{g_{s}^{2}} \int d^{10} x \sqrt{-G^{(10)}}\left(R^{(10)}+\frac{1}{M_{s}^{2}}\left(F^{2}+R^{2}\right)+\cdots+O\left(g_{s}^{2}\right)\right),
$$

where $G^{(d)}$ and $R^{(d)}$ denote $d$ dimensional metric and scalar curvature, respectively, $M_{s}$ is the string scale and $g_{s}$ is the string coupling. The explicit terms in the effective Lagrangian, as well as the "...", ${ }^{3}$ arise from tree level contributions in the perturbative string expansion, while the $O\left(g_{s}^{2}\right)$ indicates higher order string loop corrections. The term $R^{2}$ is suppressed by a factor $R / M_{s}^{2}$ relative to the Einstein term, and hence can be ignored as long as the curvature is much smaller then $1 / l_{s}^{2}$.

Compactifying to four dimensions, one obtains:

$$
\begin{aligned}
S_{\text {eff }}^{(4)} & \sim \frac{M_{s}^{8}}{g_{s}^{2}} \int_{\mathcal{M}_{6}} d^{6} x \sqrt{G^{(6)}} \int d^{4} x \sqrt{-G^{(4)}}\left(R^{(4)}+\frac{F^{2}}{M_{s}^{2}}+\cdots+O\left(g_{s}^{2}\right)\right) \\
& =\frac{M_{s}^{8}}{g_{s}^{2}} V_{6} \int d^{4} x \sqrt{-G^{(4)}}\left(R^{(4)}+\frac{F^{2}}{M_{s}^{2}}+\cdots+O\left(g_{s}^{2}\right)\right) \\
& =\int d^{4} x \sqrt{-G^{(4)}}\left(M_{P}^{2} R^{(4)}+\frac{M_{P}^{2}}{M_{s}^{2}} F^{2}+\cdots+O\left(g_{s}^{2}\right)\right) .
\end{aligned}
$$

Therefore, as in any other ten dimensional string theory compactified to four dimensions on a six dimensional manifold $\mathcal{M}_{6}$ whose volume is $V_{6}=\int_{\mathcal{M}_{6}} d^{6} x \sqrt{G^{(6)}}$, the four dimensional Planck mass $M_{P}$ is given in terms of the string scale $M_{s}$ and the string coupling $g_{s}$ by:

$$
M_{P}^{2}=\frac{M_{s}^{8}}{g_{s}^{2}} V_{6}
$$

For simplicity, in the following we set all compactification scales $M_{c} \sim 1 / R_{c}$ at the same value: $V_{6} \sim R_{c}^{6}$.

From the effective action in Eq. $(\overline{2} .2 i)$ we see that the four dimensional gauge couplings behaves like $g_{Y M} \sim M_{s} / M_{P}$. Since in the Standard Model (SM) the gauge couplings are not much smaller then 1 , we are forced to set the string scale only slightly smaller than the Planck scale. If we also take the compactification scale to be at the string scale, Eq. (2.). implies that the string coupling is of order 1 . Therefore, in such a framework we have:

$$
1985-95: \quad g_{s} \sim 1, \quad M_{s}, M_{c} \sim M_{P} .
$$

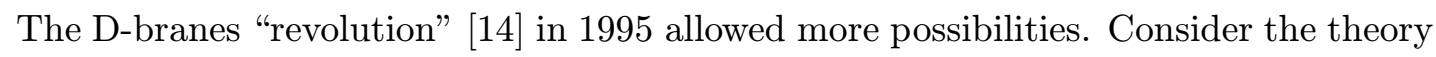
on D3-branes with the six dimensional transverse space compactified as above ${ }^{4}$. Gauge

\footnotetext{
${ }^{3}$ Actually, the string coupling is the VEV of a scalar field - the dilaton $-g_{s}=e^{\Phi}$. Hence, the "..." also include a kinetic term for $\Phi$.

${ }^{4}$ To be more precise, we discuss type II in the presence of orientifolds or, equivalently, type I strings.
} 
interactions are due to the endpoints of open strings stretched between the D-branes, and are thus confined to the $1+3$ dimensional worldvolume of the branes. The gauge couplings depend on the string coupling $g_{Y M}^{2} \sim g_{s}$ and, therefore, the string coupling cannot be much smaller than 1. An interesting framework is one where the string scale is set at the electro-weak scale, in which case the correct gravitational strength $G_{\text {Newton }} \sim M_{P}^{-2}$ is due

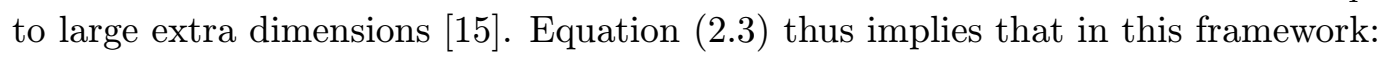

$$
\text { 1998: } \quad g_{s} \sim 1, \quad M_{s} \sim T e V, \quad M_{c} \sim 10^{-5} M_{s} .
$$

Such a framework has dramatic consequences, discussed in detail in this conference. At low energy $E<M_{s}$ we have the "SM," coupled at a TeV to the full string theory, including

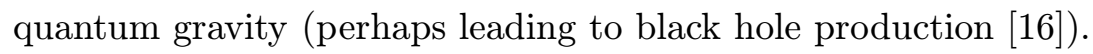

The common property to both frameworks above is that the gauge couplings depend on the string coupling, hence requiring to deal with string theories whose coupling is not much smaller than 1 . Next, I will discuss a very different possibility, to my opinion equally

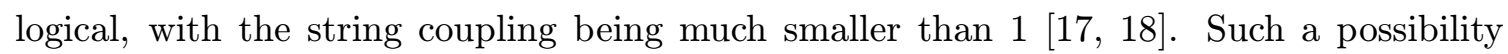
has significantly different consequences regarding many aspects [1] study. The intermediate spectrum between the electro-weak scale and the Planck scale has some unique properties. Moreover, important questions and problems get an intriguing new perspective within this framework. Examples are the issue of a runaway dilaton, or more generally, the cosmological evolution in the presence of light scalar fields. This might be done within the present experimental bounds on the time dependence of the natural constants and violation of the (weak) Equivalence Principle (EP violation), with potential predictions that can be relevant for near future experiments, like the relativistic gyroscope (or Gravity Probe B) and the satellite experiment STEP [1] $\left.\overline{9}_{1}^{\overline{1}}\right]$, which will test the principle of equivalence to at least one part in $10^{18}$. Also, the cosmological constant "double" problem - an extremely small value, yet not 0 - gets a rather interesting twist in this framework. So let me discuss this possibility, which I shall refer to as "LST at a TeV" (or strings without

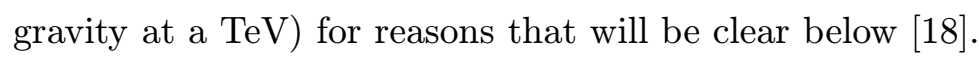

Consider string theory ${ }^{5}$ on $R^{1,3} \times \mathcal{M}_{6}$, with a small string coupling $g_{s} \ll 1$, and let us set the compactification scale of $\mathcal{M}_{6}$ at its most natural value, the string scale: $M_{c} \sim M_{s}$. The lowest allowed value which is manifestly compatible with present experiments is, as before, $M_{s} \sim \mathrm{TeV}$. Equation (2, $\left.\overline{3}^{3}\right)$ now implies ${ }^{6}$ :

$$
g_{s} \sim \frac{M_{s}}{M_{P}} \sim 10^{-16}, \quad M_{s} \sim M_{c} \sim T e V .
$$

Two immediate questions arise: how do we get the correct $g_{Y M} \sim 1$ ? and how do we get light charged SM particles?

The answers to both questions are related: charged degrees of freedom are due to Dbranes wrapped on small cycles in the compact space $\mathcal{M}_{6}$, and they couple to each other with a strength that depends only on the geometric data and not on $g_{s}$. To get light charged

\footnotetext{
${ }^{5}$ To be precise, a Type II string theory.

${ }^{6}$ Taking factors of $2 \pi$ carefully one actually obtains $g_{s} \sim 10^{-14}$.
} 
degrees of freedom requires to have small cycles, since the mass of a D-brane wrapped on a cycle with size $v$ behaves like $v / g_{s}$. Namely, the six dimensional compact space is in the vicinity of a point where it develops a singularity. The singularity is due to, say, a two sphere shrinking to a small size.

This theory is equivalent ${ }^{7}$ to a theory of an NS5-brane wrapped on a two cycle in $\mathcal{M}_{6}$. In the latter picture, as in the previous section, charged degrees of freedom are due to D-branes ending on the fivebrane. Their low lying degrees of freedom describe a gauge theory with the gauge coupling depending on the geometry of the two cycle on which the fivebrane is wrapped. For instance, if this two cycle is a two torus with radii $R_{1}, R_{2}$, the gauge coupling ${ }^{8}$ is $g_{Y M}^{2} \sim R_{1} / R_{2}$. Contrary to Eq. (글.2i $)$, the four dimensional effective action in this case is of the form:

$$
S_{e f f, l s t} \sim \int d^{4} x \sqrt{-G^{(4)}}\left(M_{P}^{2} R^{(4)}+\frac{R_{2}}{R_{1}} F^{2}+\cdots+O\left(g_{s}\right)\right) .
$$

It is thus possible to set the gauge couplings at their observed values, while having a very small string coupling.

The theory we were led to is a theory of NS5-branes with a very small string coupling. Hence, for energies well below the Planck scale we have a Little String Theory, as described in the previous section, with a scale $M_{s} \sim \mathrm{TeV}$. Namely, an LST at a TeV, as advertised.

The $\mathrm{TeV}$ signatures in this framework are unique [i] $\overline{8}_{1}^{1}$ : low lying charged SM particles are coupled to neutral string excitations without gravity. The tension of these little strings is $\sim \mathrm{TeV}^{2}$, thus predicting Regge trajectories at the electro-weak scale ${ }^{9}$. Quantum gravity effects will show up at a much higher energy. In addition, there are Kaluza-Klein (KK) and winding excitations above a $\mathrm{TeV}$, signaling $\mathrm{TeV}^{-1}$ extra dimensions, whom precise structure is model dependent.

\section{Acknowledgments:}

I am grateful to I. Antoniadis, S. Dimopoulos and D. Kutasov for collaboration on several topics presented in this talk. This work is supported in part by the European RTN network HPRN-CT-2000-00122, the BSF - American-Israel Bi-National Science Foundation, the Israel Academy of Sciences and Humanities - Centers of Excellence Program, and the German-Israel Bi-National Science Foundation.

\section{References}

[1] For a review, see M. B. Green, J. H. Schwarz and E. Witten, "Superstring Theory. Vol. 1: Introduction," "Vol. 2: Loop Amplitudes, Anomalies And Phenomenology," Cambridge, Uk: Univ. Pr. (1987) ( Cambridge Monographs On Mathematical Physics).

\footnotetext{
${ }^{7}$ by a T-duality

${ }^{8}$ in type IIA

${ }^{9}$ To compute the precise coupling of light charged particles to little string excitations, such as the excitations of the photon, require to understand the physics of D-branes near the tip of the cigar, and the way they couple to closed strings, perhaps along the lines of the program initiated in 20.20.
} 
[2] For a review, see J. Polchinski, "String theory. Vol. 1: An introduction to the bosonic string," "Vol. 2: Superstring theory and beyond," Cambridge, UK: Univ. Pr. (1998).

[3] Transparencies of the talk, http://ion.elte.hu/hep2001/scan.html, Tuesday 17.

[4] For a review, see A. Giveon and D. Kutasov, "Brane dynamics and gauge theory," Rev. Mod. Phys. 71 (1999) 983 [arXiv:hep-th/9802067].

[5] N. Seiberg, "New theories in six dimensions and matrix description of M-theory on $\mathrm{T}^{* *} 5$ and T**5/Z(2)," Phys. Lett. B 408 (1997) 98 [arXiv:hep-th/9705221].

[6] M. Berkooz, M. Rozali and N. Seiberg, "On transverse fivebranes in M(atrix) theory on T**5," Phys. Lett. B 408 (1997) 105 [arXiv:hep-th/9704089].

[7] For a review, see O. Aharony, "A brief review of 'little string theories'," Class. Quant. Grav. 17 (2000) 929 [arXiv:hep-th/9911147].

[8] For a review, see O. Aharony, S. S. Gubser, J. Maldacena, H. Ooguri and Y. Oz, "Large N field theories, string theory and gravity," Phys. Rept. 323 (2000) 183 [arXiv:hep-th/9905111].

[9] J. Maldacena, "The large $N$ limit of superconformal field theories and supergravity," Adv. Theor. Math. Phys. 2 (1998) 231 [Int. J. Theor. Phys. 38 (1998) 1113] [arXiv:hep-th/9711200].

[10] O. Aharony, M. Berkooz, D. Kutasov and N. Seiberg, "Linear dilatons, NS5-branes and holography," JHEP 9810 (1998) 004 [arXiv:hep-th/9808149].

[11] A. Giveon and D. Kutasov, "Little string theory in a double scaling limit," JHEP 9910 (1999) 034 [arXiv:hep-th/9909110]; "Comments on double scaled little string theory," JHEP 0001 (2000) 023 [arXiv:hep-th/9911039].

[12] S. Elitzur, A. Giveon and D. Kutasov, "Branes and $N=1$ duality in string theory," Phys. Lett. B 400 (1997) 269 [arXiv:hep-th/9702014].

[13] N. Seiberg, "Electric - magnetic duality in supersymmetric nonAbelian gauge theories," Nucl. Phys. B 435 (1995) 129 [arXiv:hep-th/9411149].

[14] J. Polchinski, "Dirichlet-Branes and Ramond-Ramond Charges," Phys. Rev. Lett. 75 (1995) 4724 [arXiv:hep-th/9510017].

[15] N. Arkani-Hamed, S. Dimopoulos and G. R. Dvali, "The hierarchy problem and new dimensions at a millimeter," Phys. Lett. B 429 (1998) 263 [arXiv:hep-ph/9803315];

I. Antoniadis, N. Arkani-Hamed, S. Dimopoulos and G. R. Dvali, "New dimensions at a millimeter to a Fermi and superstrings at a TeV," Phys. Lett. B 436 (1998) 257 [arXiv:hep-ph/9804398].

[16] S. Dimopoulos and G. Landsberg, "Black holes at the LHC," Phys. Rev. Lett. 87 (2001) 161602 [arXiv:hep-ph/0106295].

[17] I. Antoniadis and B. Pioline, "Low-scale closed strings and their duals," Nucl. Phys. B 550 (1999) 41 [arXiv:hep-th/9902055].

[18] I. Antoniadis, S. Dimopoulos and A. Giveon, "Little string theory at a TeV," JHEP 0105 (2001) 055 [arXiv:hep-th/0103033].

[19] For a review, see T. Damour, "Questioning the equivalence principle," arXiv:gr-qc/0109063.

[20] A. Giveon and D. Kutasov, "Notes on $\operatorname{AdS}(3)$," arXiv:hep-th/0106004; A. Giveon,

D. Kutasov and A. Schwimmer, "Comments on D-branes in AdS(3)," arXiv:hep-th/0106005. 